\title{
Federal medical biological agency of Russia's efforts to support Russian athletes during COVID-19 outbreak
}

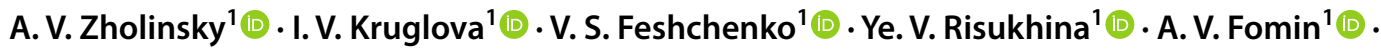

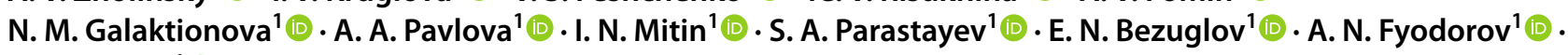 \\ S. I. Barshak ${ }^{1}$ (])
}

Received: 1 June 2021 / Accepted: 16 October 2021 / Published online: 6 November 2021

(c) The Author(s), under exclusive licence to Springer-Verlag Italia S.r.l., part of Springer Nature 2021

\begin{abstract}
This paper dwells upon COVID-19-related efforts of the Center for Sports Medicine, Federal Medical and Biological Agency of Russia. The Agency has the following precautions in place: regular polymerase chain reaction (PCR) testing of athletes and staff; double PCR testing before going to training camps or medical examinations; isolating athletes and their traceable contacts when COVID is suspected; observation and isolation wards set up at training camp venues. Athlete vaccination has begun. Athletes are provided online advice on health, diet, and exercising plus special care for chronically ill athletes and remote psychological counseling. Athletes recovering from COVID-19 are offered rehabilitation programs and doctorsupervised return to training. Specialists of the Research Department at FMBA's Center for Sports Medicine carried out a research dedicated to the prevalence of COVID-19 and different variants of its course in Russian athletes. The study period lasted from March to December 2020. A total of 27,438 records were analyzed. In May, June, July and August 2020, the percentage of positive PCR tests for athletes was significantly lower than the nationwide percentage at $p<0.05$, Pearson's chi-squared test. However, the differences were nullified by September-October. The disease was mild or asymptomatic in most patients. Athletes of summer sports were found to be most likely to contract COVID-19.
\end{abstract}

Keywords COVID-19 $\cdot$ International competitions $\cdot$ Rehabilitation $\cdot$ Prevention $\cdot$ Medical care $\cdot$ Psychological counseling

\section{Introduction}

Early 2020 saw the emergence of highly unusual circumstances no one could predict as the novel coronavirus was spreading across the world and soon developed into a

S. I. Barshak

sergeybarshak@gmail.com

A. V. Zholinsky

fnkcsm@sportfmba.ru

I. V. Kruglova

KruglovaIV@sportfmba.ru

V. S. Feshchenko

vfmed@yandex.ru

Ye. V. Risukhina

RisuhinaEV@sportfmba.ru

A. V. Fomin

FominAV@ @portfmba.ru

N. M. Galaktionova omposskrf@sportfmba.ru pandemic. December 31, 2019, Chinese health authorities reported to WHO on a case of pneumonia of unknown etiology that had been discovered in Wuhan [1]. In January 2020, Chinese scientists found out this new pneumonia was caused by 2019-nCoV coronavirus (SARS-CoV-2). SARS-CoV-2

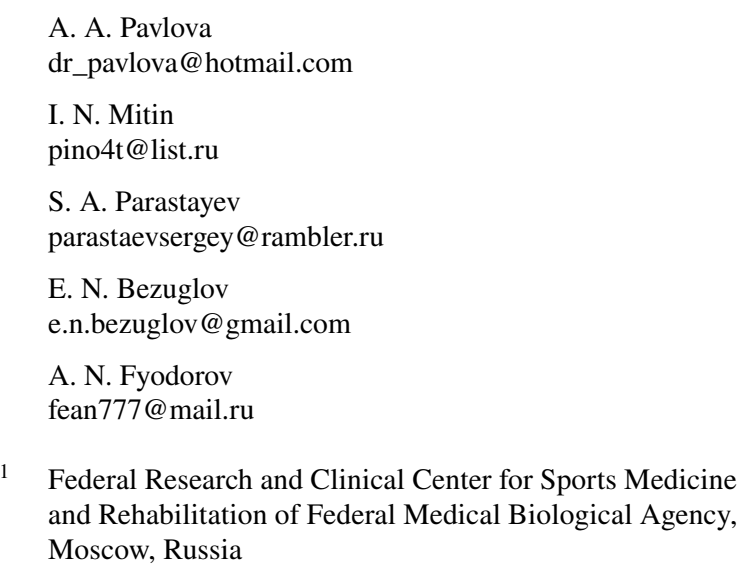

1 Federal Research and Clinical Center for Sports Medicine and Rehabilitation of Federal Medical Biological Agency, Moscow, Russia 
was a novel strain that had not been identified in humans before [2].

In early February 2020, the Health Ministry of Russia approved its first revision of Novel Coronavirus Prevention, Diagnosis, and Treatment Guidelines [3].

Many sporting events had to be cancelled, including the 2020 Tokyo Olympics [4]. Russia canceled all of its competitions, training camps, and closed all federal training camp venues [5]. Russia's sports lockdown lasted from March 29 until May 25 2020. In early July, the Ministry of Sport resumed hosting national and international competitions in the country [6].

\section{Quarantine and PCR testing}

Two safety directives were made to avoid spreading of the infection among athletes. First, the infection shall not enter venues; second, athletes shall not infect each other within the team. Multiple interconnected measures were put in place, including polymerase chain reaction (PCR) testing, monitored quarantine in single rooms at the training centers until test results are available, and restricted access to training camp venues. Athletes would only start training once a negative PCR test was provided [7].

Safety guidelines developed by Russian specialists are similar to those of their international counterparts. They all underline the importance of double PCR testing before training camps and regular testing as well [8]. Sport scientists and doctors all around the world are considering various options to resume training. A 'quarantined' training camp allows athletes to continue training without major risk of getting infected [9]. International experts recommend different quarantine duration, usually from 8-10 to 14 days. One study on athletes showed the infection probability drop from $27 \%$ on Day 5 of the camp to $<5 \%$ on Day 10 [10]. Some papers highlight successful cases of resuming training and matches enabled by strict hygiene measures including regular PCR testing [11]. Research on 26 football teams in Denmark showed a $0.53 \%$ occurrence rate of positive SARSCoV-2 test results [12]. Some studies report that in certain sports, airborne transmission of SARS-CoV-2 is rather unlikely [13].

FMBA of Russia began PCR-testing of athletes on a large scale as soon as training camps resumed in late May 2020 [14]. The testing facilities were set up at FMBA clinics and training camps. A total of 27,438 tests were taken in MarchDecember 2020.

To minimize the risk of infection, athletes and staff would take PCR tests twice within a minimum 24-h window before going to training camps. They also had to stay isolated in their rooms at the training centers for 2 weeks after arriving. Athletes could start training once two negative PCR tests taken in their rooms during the isolation period were provided.

Athletes and staff were subject to weekly PCR testing while staying at the camps. Individuals showing acute respiratory infection symptoms were bound to take additional PCR tests. Two-stage PCR testing was also required before advanced medical examinations that Russian athletes are obliged to undergo twice a year.

Infected persons were subject to immediate isolation or hospitalization if necessary. Should an athlete test positive during a training camp, infected persons and their contacts would cease training immediately; athletes would then be placed under continuous monitoring in the isolation wards set up at the training camp venue. Training would be resumed after 2 weeks in isolation provided a negative PCR test. Negative-tested athletes would continue training under daily medical monitoring including regular temperature checks, wearing masks and respecting the social distance.

\section{Preventive measures}

During the lockdown that resulted in an unprecedented suspension of training, sports medicine and rehabilitation experts continued to provide advisory assistance to athletes remotely on a daily basis. Researchers of the Federal Research and Clinical Center of Sports Medicine and Rehabilitation of FMBA prepared guidelines on exercising and diet in pandemic; these materials were distributed among doctors and athletes. The recommendations are based on the proposals of the European College of Sports Science (ECSS) [15]. Special care was provided to chronically ill athletes. Training videos on physical therapy were prepared to help them stay in shape. Doctoral advice was provided remotely; besides, the athletes were following individual recovery programs under medical observation.

Multiple restrictions, unexpected changes in lifestyle and the inability to follow the usual schedule could not but affect the athletes' psyche [16]. Pandemic-related situation and social isolation could catalyze multiple mental issues including stress disorders, irritability, sleep disturbances, mood disorders, financial worries, boredom, loneliness, and lack of communication [17-21].

Specialists of the Medical and Psychological Care Department, Center for Sports Medicine, have been providing 24/7 support to athletes since the pandemic began. The advice was provided remotely either on an individual basis or in groups using video conferencing and online meetings. For remote counseling, specialists drew upon the recommendations of their Chinese peers who had been the first to face the coronavirus [22, 23].

Six hundred and forty-four athletes used such remote counseling in over 8000 sessions. Remote psychological assistance is provided to relieve psycho-emotional stresses, 
mitigate vegetative reactions, restore sleep, and help adapt to uncertainty life. Athletes were also trained self-regulation skills such as breath control, muscle tone control, and use of images. Webinars on such topics as confidence, self-support, fear and anxiety, and psychological work towards goals were held on a regular basis. Center's doctors received guidelines on information support and counseling for athletes, including emotional burnout prevention and self-regulation of psycho-emotional state.

\section{Vaccination of athletes}

As of writing this paper, the Center for Sports Medicine is starting to vaccinate athletes of the Russian national teams against SARS-CoV2. Vaccination is regulated under the Chief Medical Officer's Order No. 41 dd. December 3, 2020, and FMBA Order No. 227 dd. August 21, 2020. These measures are in line with the IOC's position on vaccinating athletes in the run-up to the upcoming Olympic Games in Tokyo [24]. The Russian Federation has registered two COVID-19 vaccines for adults aged 18-60 that induce humoral and cellular immunity to SARS-CoV2:

- Combined vector vaccine (Gam-COVID-Vac) registered on August 11, 2020,

- Peptide antigen-based vaccine (EpiVacCoron) registered on October 13, 2020.

\section{Therapy and rehabilitation}

Mild COVID-19 survivors among athletes were treated at home or in the isolation wards of training camp venues should their infection be detected during a training camp. Severe forms were treated in inpatient facilities. Inpatient treatment used a combination of antiviral and antibacterial medicines recommended by the Ministry of Health [3]. Anticoagulants and detoxification medicines were prescribed in severe cases. No WADA-monitored drugs were used.

Athletes who contracted COVID-19 were actively followed up after recovery. According to the recommendations of international experts, these athletes were undergoing pulmonary and heart tests [25]. Additional treatment and rehabilitation was assigned when necessary. As far as post-COVID return to sports is concerned, the recommendations of British specialists were taken into account. Their guide applies to athletes who had mild or moderate COVID-19. It outlines a six-step recovery routine with the last step being the return to competitive sports [26]. The sports resumption protocols must be adjusted for the fact that an athlete's condition may deteriorate within the first few weeks of resuming high-intensity training [27].

\section{Methods}

Specialists of the Research Department at FMBA's Center for Sports Medicine carried out a research dedicated to the prevalence of COVID-19 and variants of its course in Russian athletes. A total of 27,438 records were analyzed. The study period lasted from March to December 2020. The total number of PCR tests taken by athletes and the number of positive tests with breakdown by month was analyzed. These figures were then compared against the nationwide general statistics. Statistical differences were detected by Pearson's Chi-squared test. The difference was deemed significant at $p<0.05$. The Center also analyzed the discharge summaries of hospitalized athletes. Statistical data were broken down by sex and age, duration of hospital stay, course of the disease, and lung damage severity. Occurrence rates of the most common symptoms were also calculated.

\section{Results}

PCR testing rate grew in proportion to the number of athletes involved in training and competitions and peaked from September to October. As more and more PCR tests were taken, the number of positives rose as well, peaking in October when 224 athletes tested positive, see Fig. 1.

In May and June 2020, the percentage of positive PCR tests for athletes differed significantly from the nationwide statistics at $p<0.01$, Pearson's chi-squared test. In July, August and September the difference was significant at $p<0.05$ [28]. However, the differences were nullified by September-October, see Fig. 2.

A total of 609 athletes tested positive for SARS-COV-2 by December 31; traced contacts totaled 1508 persons.

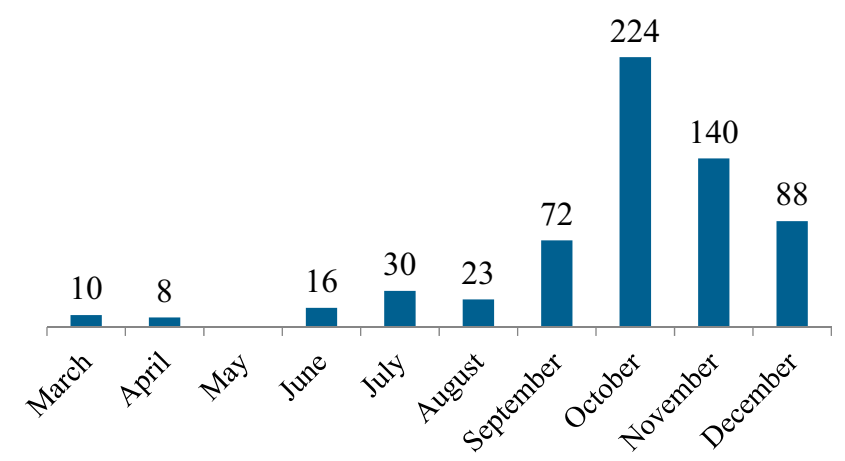

Fig. 1 Monthly distribution of national teams' athletes tested positive, 2020 
Fig. 2 Positive PCR test results in $\%$ to the total tests taken.

*Significance at $p<0.01$, Pearson's chi-squared test. $* *$ Significance at $p<0.05$, Pearson's chi-squared test

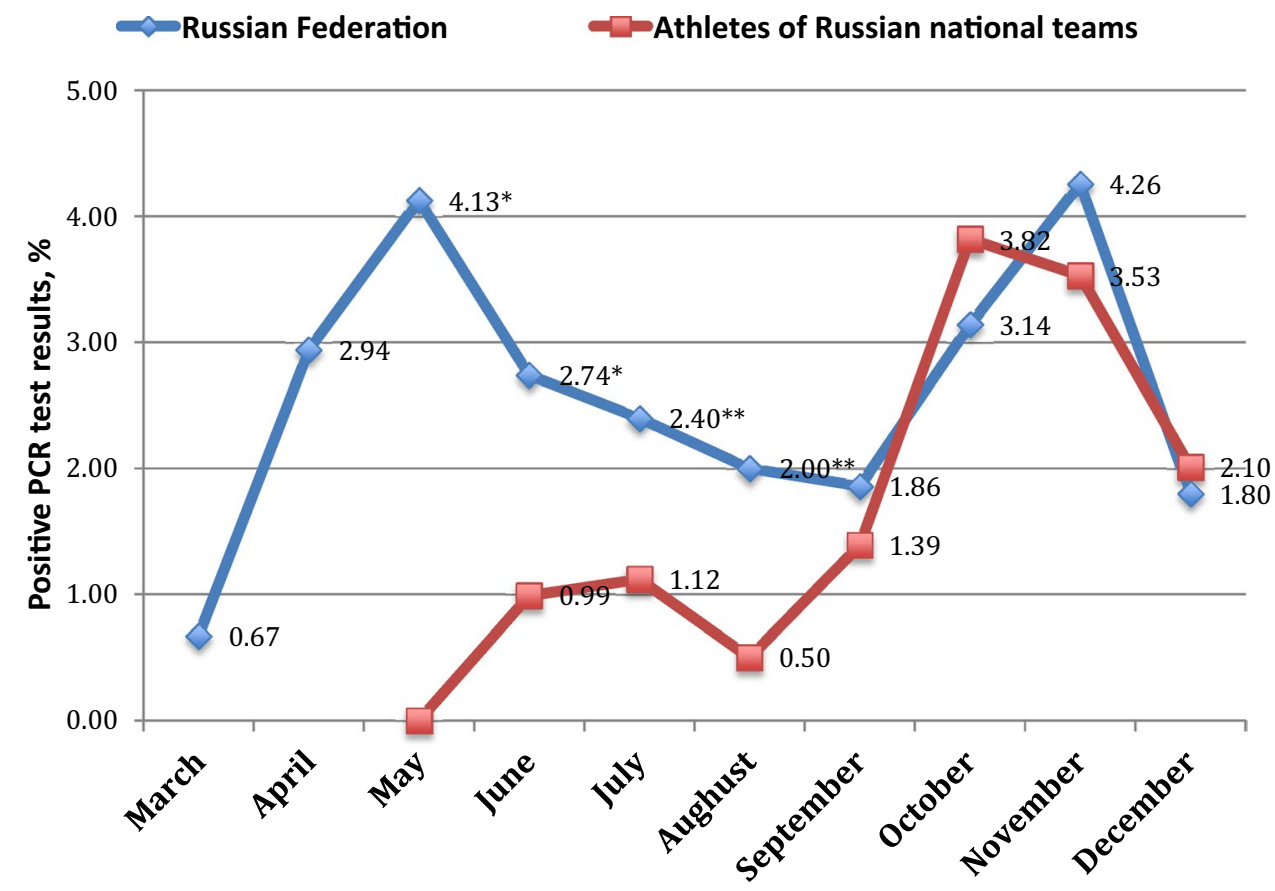

In 58\% ( $n=335)$, COVID-19 was found in testing before training camps. The rest of the positive tests were distributed as follows: additional testing during training camp $(4.6 \%, n=28)$; testing before medical examinations, hospitalizations, and rehabilitation at FMBA clinics $(11 \%, n=67)$; testing before advanced medical examinations $(16 \%, n=96)$, self-requested testing outside FMBA facilities $(4.4 \%, n=27)$. The remaining 36 positive tests were taken in other contexts including pre-competition testing, mandatory pre-travel testing, or in cases of ARI symptoms.

Nearly three-quarters of the athletes who tested positive had a symptomless or mild course. They were placed in isolation at home $(54 \%, n=328)$ or in at-venue wards $(46 \%$, $n=281$ ).

Infected athletes were aged 22 on average, the oldest being 49 and the youngest being 12 . Men were $35 \%$ more likely to test positive ( $42 \%$ were women, $n=256 ; 58 \%$ were men, $n=353$ ).

Athletes representing summer sports had the most infections: $n=39$ in box, $n=40$ in judo, $n=27$ in taekwondo, $n=26$ in rhythmic gymnastics, $n=23$ in rugby.

Although most of the affected young persons only have asymptomatic or mild COVID-19, 14\% of the athletes required hospitalization $(n=85)$, see Fig. 3 .

A part of the hospitalized athletes $(80 \%)$ had a mild course $(n=68)$. The moderate-to-severe course was observed in $18 \%$ of all cases, $n=15$; the severe course was observed in $2 \%, n=2$. These athletes had pulmonary tissue alterations on CT scans. CT1 lung damage $(<25 \%$, mild pneumonia) was reported for five athletes; CT2 damage (25\% to

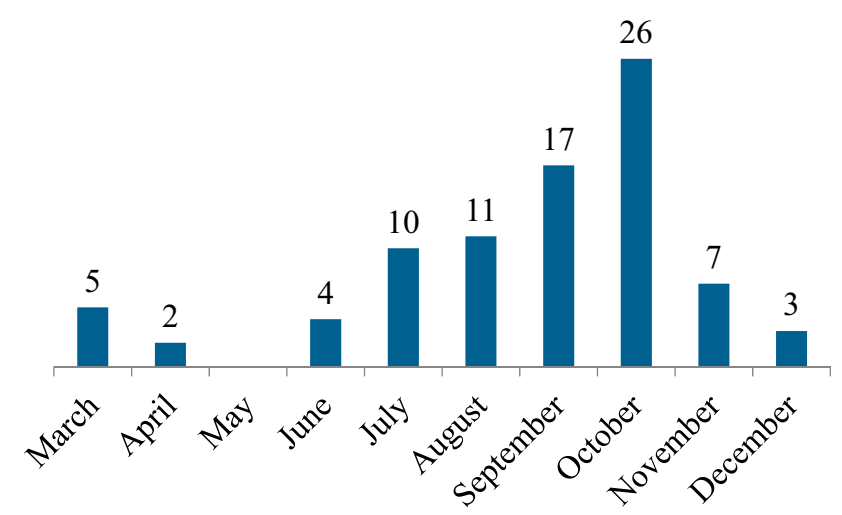

Fig. 3 Athletes hospitalized with SARS-CoV2 in 2020, breakdown by months

$50 \%$, moderate pneumonia) was reported for 2 . No cases of CT3 (moderate-to-severe pneumonia, $50 \%$ to $75 \%$ ) or CT4 (severe pneumonia, $>75 \%$ ).

Almost twice as many men were hospitalized as women (63.5\%, $n=54$, cf. 36.5\%, $n=31$ ); hospitalized athletes were aged $24 \pm 4.2$ on average. Hospital stay lasted 10 days on average, 22 days at max.

Clinical signs were observed in $90 \%(n=73)$ of the hospitalized athletes, most commonly loss of smell and/or taste, anemia, nasal congestion, shortness of breath, and sore throat, see Fig. 4. 


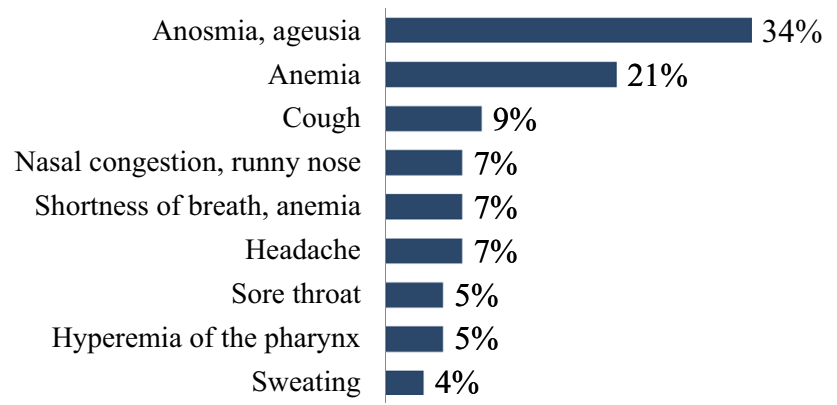

Fig. 4 Symptoms in the hospitalized athletes

\section{Discussion}

The number of athletes who contracted COVID-19 was relatively low. In most infected athletes disease course was mild or asymptomatic. Initially, infection rate amongst Russian athletes was significantly lower than in general population due to strict quarantine measures. Once active training and competitive involvement resumed, the percentage of positive tests in athletes started to grow and it did not differ significantly from nationwide statistics as of September-October 2020. The number of athletes tested positive during training camps was low; most of the athletes who contracted COVID19 got infected outside training camps, e.g., at home, in public transport, etc. In means that "quarantine" training camp with regular PCR testing is a rather effective way to maintain sport-specific training during the pandemic.

Despite relatively low prevalence of severe symptoms it is crucial to monitor COVID-19 survivors for possible delayed symptoms and long-term complications. For instance, one assumption is that similarly to influenza, COVID-19 might have cardiovascular complications. Myocardial damage marked by an increase in circulating cardiac troponin has been reported in $28 \%$ of severe cases [29]. Prevalence and clinical consequences remain unknown with respect to mild/ asymptomatic patients. There are concerns that this disease may uniquely affect athletes who return to training and competing [30]. Studies on athletes who had mild COVID-19 have rather contradictory results, as some researchers report mild cardiovascular symptoms whilst others state a lack of statistically significant differences between healthy subjects and COVID-19 survivors [31-33].

Therefore, the latter must be subject to follow-up to detect and treat possible long-term effects as well as to develop methods to address such effects in the most effective manner. Future priorities include the development of functional testing programs for athletes who had COVID-19 to help them safely return to training and competitions; and the development of guidelines for teams' doctors on the prevention and treatment of the novel coronavirus infection.
The pandemic began slightly over a year ago; and yet, despite the sheer number of COVID-19 papers, research on professional athletes remains limited. The existing guidelines are mainly based on expert opinions and consensus [34]. Sports science is yet to come up with a commonly accepted protocol on return to sports in a pandemic.

\section{Conclusion}

Specialists of FMBA's Center for Sports Medicine managed to adapt medical care for athletes to the conditions of the pandemic. Over time, athletes were able to resume their training whilst taking precautions, which prevented an infection catastrophe; these precautions were based on international practices among other things. Extensive preventive measures are in place. Survivors are provided rehabilitative care to help return to training and competitions.

Most athletes of Russia's national teams were asymptomatic, and most hospitalized athletes only had mild COVID-19 without lung damage. Once active training and competitive involvement resumed, the percentage of positive tests in athletes did not differ significantly from nationwide statistics.

Funding The authors received no financial support for the research, authorship, and/or publication of this article.

\section{Declarations}

Conflict of interest The authors have no conflicts of interest to declare. All co-authors have seen and agree with the contents of the manuscript and there is no financial interest to report. We certify that the submission is original work and is not under review at any other publication.

Ethical approval This is a retrospective study. The data used in this study were collected by the clinics of Federal Medical and Biological Agency of Russia in accordance with Russian legislation on protection of personal data and provided to us in anonymized format. Nationwide statistics were obtained from open sources. According to the legislation of the Russian Federation, retrospective studies using anonymized data do not require additional ethical approval.

Informed consent Retrospective studies using anonymized data do not require informed consent.

\section{References}

1. WHO (2020) Pneumonia of unknown cause China. Disease outbreak news. URL: https://www.who.int/csr/don/05-janua ry-2020-pneumonia-of-unkown-cause-china/en/. Accessed 11 Mar 2021 
2. Novel Coronavirus (2020) China disease outbreak news: Update 12 January 2020. URL: https://www.who.int/csr/don/12-janua ry-2020-novel-coronavirus-china/en. Accessed 11 Mar 2021

3. Prevention, diagnosis, and treatment of the novel coronavirus infection (COVID-19): temporary guidelines [in Russia] URL: https://minzdrav.gov.ru/ministry/med_covid19. Accessed 11 Mar 2021

4. Joint statement from the international Olympic committee and the Tokyo 2020 organising committee. URL: https://www. olympic.org/news/joint-statement-from-the-internationalolympic-committee-and-the-tokyo-2020-organising-committee. Accessed 11 Mar 2021

5. Ministry of Sport Order No. 216 dated March 16, 2020 on the cancellation or postponement of sporting events in the russian federation for COVID-19 prevention [in Russia]. URL: http://www.consultant.ru/document/cons_doc_LAW_348117/. Accessed 11 Mar 2021

6. Ministry of Sport Order No. 497 dated July 8, 2020 on hosting sporting events in the russian federation [in Russia] URL: https://minsport.gov.ru/documents/ministry-orders/35056/. Accessed 11 Mar 2021

7. Temporary procedures for organizing and hosting training camps at Ministry of Sport's Federal Training Camp Venues (approved by the Ministry of Sport, Rev. February 20, 2021) [in Russia]. URL: http://www.consultant.ru/document/cons_doc_ LAW 357961/. Accessed 11 Mar 2021

8. Rankin A, Massey A, Falvey ÉC et al (2021) Infographic. COVID-19 RT-PCR testing for elite athletes. Br J Sports Med. https://doi.org/10.1136/bjsports-2020-103751

9. Washif JA, Mohd Kassim SFA, Lew PCF, Chong CSM, James C (2021) Athlete's perceptions of a "quarantine" training camp during the COVID-19 lockdown. Front Sports Act Living 2:622858. https://doi.org/10.3389/fspor.2020.622858 (PMID :33521634;PMCID:PMC7841328)

10. Atherstone C, Peterson ML, Malone M, Honein MA, MacNeil A, O'Neal CS, Paul S, Harmon KG, Goerl K, Wolfe CR, Casani J, Barrios LC (2021) COVID-19 collegiate athlete testing group time from start of quarantine to SARS-CoV-2 positive test among quarantined college and university athletes-17 states, June-October 2020. Morb Mortal Wkly Rep 70(1):7-11. https://doi.org/10.15585/mmwr.mm7001a2 (PMID: 33417591; PMCID: PMC7790154)

11. Meyer T, Mack D, Donde K, Harzer O, Krutsch W, Rössler A, Kimpel J, von Laer D, Gärtner BC (2021) Successful return to professional men's football (soccer) competition after the COVID-19 shutdown: a cohort study in the German Bundesliga. Br J Sports Med 55(1):62-66. https://doi.org/10.1136/bjsports2020-103150 (Epub 2020 Sep 24. PMID: 32972979; PMCID: PMC7788215)

12. Pedersen L, Lindberg J, Lind RR, Rasmusen H (2021) Reopening elite sport during the COVID-19 pandemic: experiences from a controlled return to elite football in Denmark. Scand J Med Sci Sports. https://doi.org/10.1111/sms.13915 (Epub ahead of print. PMID: 33480037)

13. England R, Peirce N, Wedatilake T, Torresi J, Kemp S, Cook M, Mitchell S, Harland A (2021) The potential for airborne transmission of SARS-CoV-2 in sport: a cricket case study. Int J Sports Med. https://doi.org/10.1055/a-1342-8071 (Epub ahead of print. PMID: 33511617)

14. Ministry of Sport Order No. 394/1 dated May 25, 2020 on the resumption of training at ministry of sport-governed organizations in light of the need to prevent the spread of the novel coronavirus infection (COVID-19) in the Russian Federation [in Russia] URL: https://minsport.gov.ru/documents/ministryorders/35006/. Accessed 11 Mar 2021
15. Narici M, De Vito G, Franchi M, Paoli A, Moro T, Marcolin G, Grassi B, Baldassarre G, Zuccarelli L, Biolo G, di Girolamo FG, Fiotti N, Dela F, Greenhaff P, Maganaris C (2020) Impact of sedentarism due to the COVID-19 home confinement on neuromuscular, cardiovascular and metabolic health: physiological and pathophysiological implications and recommendations for physical and nutritional countermeasures. Eur J Sport Sci. https://doi.org/10.1080/17461391.2020.1761076

16. Leguizamo F, Olmedilla A, Núñez A et al (2021) Personality, coping strategies, and mental health in high-performance athletes during confinement derived from the COVID-19 pandemic. Front Public Health 8:561198. https://doi.org/10.3389/ fpubh.2020.561198

17. Perrin PC, McCabe OL, Everly GS, Links JM (2009) Preparing for an influenza pandemic: mental health considerations. Prehosp Disaster Med 24(3):223-230. https://doi.org/10.1017/ S1049023X00006853

18. Bai Y, Lin CC, Lin CY, Chen JY, Chue CM, Chou P (2004) Survey of stress reactions among health care workers involved with the sars outbreak. Psychiatr Serv 55(9):1055-1057. https:// doi.org/10.1176/appi.ps.55.9.1055

19. Brooks SK, Webster RK, Smith LE, Woodland L, Wessely S, Greenberg N, Rubin GJ (2020) The psychological impact of quarantine and how to reduce it: rapid review of the evidence. The Lancet 395(10227):912-920. https://doi.org/10.1016/ S0140-6736(20)30460-8

20. Cava MA, Fay KE, Beanlands HJ, McCay EA, Wignall R (2005) The experience of quarantine for individuals affected by SARS in toronto. Public Health Nurs 22(5):398-406. https://doi.org/ 10.1111/j.0737-1209.2005.220504.x

21. Facer-Childs ER, Hoffman D, Tran JN, Drummond SPA, Rajaratnam SMW (2021) Sleep and mental health in athletes during COVID-19 lockdown. Sleep. https://doi.org/10.1093/sleep/ zsaa261 (Epub ahead of print. PMID: 33535229)

22. Khan S, Siddique R, Li H, Ali A, Shereen MA, Bashir N, Xue M (2020) Impact of coronavirus outbreak on psychological health. J Glob Health 10(1):010331. https://doi.org/10.7189/jogh.10. 010331

23. Liu S, Yang L, Zhang C, Xiang Y, Liu Z, Hu X, Zhang B (2020) Online mental health services in China during the COVID-19 outbreak. Lancet Psychiatry. https://doi.org/10.1016/S22150366(20)30077-8

24. IOC discusses preparations for Tokyo 2020 and Beijing 2022 with national Olympic committees URL: https://www.olympic. org/news/ioc-discusses-preparations-for-tokyo-2020-and-beiji ng-2022-with-national-olympic-committees-1. Accessed 11 Mar 2021

25. Löllgen H, Bachl N, Papadopoulou T et al (2020) Recommendations for return to sport during the SARS-CoV-2 pandemic. BMJ Open Sport Exerc Med. https://doi.org/10.1136/ bmjsem-2020-000858

26. Elliott N, Martin R, Heron N et al (2020) Infographic. Graduated return to play guidance following COVID-19 infection. Br J Sports Med 54:1174-1175. https://doi.org/10.1136/bjspo rts-2020-102637

27. Mulcahey MK, Gianakos AL, Mercurio A, Rodeo S, Sutton KM (2021) Sports medicine considerations during the COVID19 pandemic. Am J Sports Med 49(2):512-521. https://doi. org/10.1177/0363546520975186 (Epub 2020 Nov 16 PMID: 33196320 )

28. Ritchie H, Ortiz-Ospina E, Beltekian D, Mathieu E, Hasell J, Macdonald B, Giattino C, Appel C, Roser M, Yunits B, van Woerden E, Gavrilov D, Bergel M, Ahmad S, Crawford J, Gerber M. Coronavirus pandemic (COVID-19) - the data. Published online at OurWorldInData.org. Retrieved from: https:// 
ourworldindata.org/coronavirus-data. Accessed 11 Mar 2021. [Online Resource]

29. Shi S, Qin M, Shen B, Cai Y, Liu T, Yang F, Gong W, Liu X, Liang J, Zhao Q, Huang H (2020) Association of cardiac injury with mortality in hospitalized patients with COVID-19 in Wuhan, China. JAMA Cardiol 5(7):802-810

30. Baggish AD, Kim JH, Martinez MW, Prutkin JM (2020) Blog British Journal of Sports Medicine [Internet]. URL: https:// blogs.bmj.com/bjsm/2020/04/24/the-resurgence-of-sport-inthe-wake-of-covid-19-cardiac-considerations-in-competitiveathletes/

31. Małek ŁA, Marczak M, Miłosz-Wieczorek B, Konopka M, Braksator W, Drygas W, Krzywański J (2021) Cardiac involvement in consecutive elite athletes recovered from Covid-19: a magnetic resonance study. J Magn Reson Imaging. https:// doi.org/10.1002/jmri.27513 (Epub ahead of print. PMID: 33474768 )

32. Kim JH, Levine BD, Phelan D, Emery MS, Martinez MW, Chung EH, Thompson PD, Baggish AL (2021) Coronavirus disease 2019 and the athletic heart: emerging perspectives on pathology, risks, and return to play. JAMA Cardiol 6(2):219 227. https://doi.org/10.1001/jamacardio.2020.5890
33. Gervasi SF, Pengue L, Damato L, Monti R, Pradella S, Pirronti T, Bartoloni A, Epifani F, Saggese A, Cuccaro F, Bianco M, Zeppilli P, Palmieri V (2021) Is extensive cardiopulmonary screening useful in athletes with previous asymptomatic or mild SARS-CoV-2 infection? Br J Sports Med 55(1):54-61. https:// doi.org/10.1136/bjsports-2020-102789 (Epub 2020 Oct 5. PMID: 33020140; PMCID: PMC7536638)

34. Verwoert GC, de Vries ST, Bijsterveld N, Willems AR, Vd Borgh R, Jongman JK, Kemps HMC, Snoek JA, Rienks R, Jorstad HT (2020) Return to sports after COVID-19: a position paper from the Dutch sports cardiology section of the Netherlands society of cardiology. Neth Heart J 28(7-8):391-395. https://doi.org/10.1007/s12471-020-01469-z (PMID:3266205 8;PMCID:PMC7357275)

Publisher's Note Springer Nature remains neutral with regard to jurisdictional claims in published maps and institutional affiliations. 